\title{
DESARROLLO CULTURAL PREHISPÁNICO EN EL VALLE DEL ALTO UPANO: Análisis Cerámico del Sitio "La Lomita", Morona Santiago, Ecuador.
}

\author{
Estanislao Pazmiño N.P.
}

\begin{abstract}
Resumen
El presente artículo se encuentra basado en el trabajo de investigación realizado como parte de mi disertación de grado. El estudio se desarrolló a partir del análisis de la colección cerámica del sitio La Lomita en la zona del Alto Upano, amazonía ecuatoriana. La región presenta evidencia de una fuerte ocupación prehispánica que ha sido expuesta en varias investigaciones anteriores (Porras, 1985, 1987, Salazar, 1998, 1999, 2000; Rostain, 1999a, 1999b, 1999c, 2006). Aunque en principio se estableció una larga presencia del grupo cultural Upano (Porras, 1987), recientes investigaciones (Rostain, 1999b, 2006) confrontan esa información y plantean la existencia de al menos dos claras ocupaciones: Upano y Huapula. Con ello quedó expuesta la posibilidad de que en la región exista evidencia de más ocupaciones. El presente trabajo confirma la existencia de tres conjuntos cerámicos distintos registrados en el sitio La Lomita: Sangay, Upano y Huapula. La definición de estos conjuntos cerámicos, sugiere la existencia de distintos períodos de ocupación del valle en los que la interacción con la zona andina se desarrolló en diferentes escalas.
\end{abstract}

\begin{abstract}
The present article is based on an investigation that was part of my dissertation. The study developed following the analysis of the ceramics collection of La Lomita in the Alto Upano region of the Ecuadorian Amazon. The region presents evidence of a strong pre-Hispanic settlement that has been studied in various previous investigations (Porras 1985, 1987, Salazar 1995, 1998, 1999, 2000, Rostain 1999a, 1999b, 1999c, 2006.). Although at first the lengthy presence of the Upano cultural group was established (see Porras 1987), recent investigations (Rostain 1999b, 2006) confront this information and propose the existence of at least two clear settlements: Upano and Huapula. These studies exposed the possibility that there is evidence in the region of even more settlements. The present work confirms the existence of three different ceramic groupings registered in La Lomita: Sangay, Upano, and Huapula. The definition of these ceramic groupings suggests the existence of distinct periods of settlement in the valley, in which the interaction with the Andean region developed on different scales.
\end{abstract}

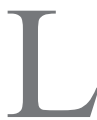
a investigación arqueológica en la región amazónica ecuatoriana es relativamente nueva en el ámbito científico. Diversos trabajos se han desarrollado en la región con interesantes aportes a la comprensión del desenvolvimiento cultural. A pesar de ello, los investigadores han enfrentado diferentes problemas, sobre todo debido a que la evidencia preservada en el registro arqueológico es limitada; por lo que gran parte de la cultura material recuperada en la amazonía corresponde a restos cerámicos. 
Las excavaciones llevadas a cabo por Ernesto Salazar entre 1995 y 1999 en el sitio de La Lomita, recuperaron abundante material cerámico que fue analizado como parte de la disertación de licenciatura presentada en junio del 2008. La única información disponible hasta ese entonces sobre estudios cerámicos en la región fue el trabajo de Pedro Porras (1987). La tipología cerámica elaborada por el mencionado autor padeció de fuertes críticas al igual que su cronología; sin embargo, ningún trabajo posterior se enfocó en la revisión y corrección del estudio de Porras.

Bajo estas circunstancias el análisis del material cerámico del sitio La Lomita se enfocó en dos objetivos centrales que fueron por un lado, el depurar la tipología estructurada por Porras por medio de la construcción de una tipología para el sitio; y por otro lado, elaborar una cronología cerámica para este sitio a partir de la tipología previamente elaborada.

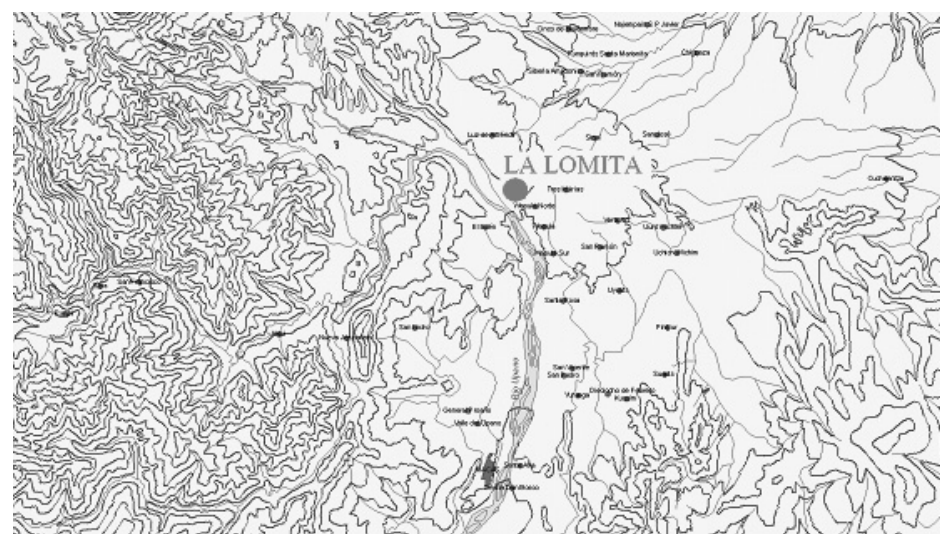

FIGURA 1.

Mapa del valle del Alto Upano indicando la ubicación del sitio La Lomita

\section{Geografía de la zona de estudio}

El sitio La Lomita forma parte del complejo arqueológico Huapula, ubicado en el valle del Alto Upano, provincia de Morona Santiago. Limitado al Oeste por la cordillera Real Andina y al Este por la cordillera del Cutucú, el valle forma una especie de llanura cortada abruptamente por el cauce del río Upano. Éste tras miles de años ha dado origen a un espectacular cañón, que en algunos tramos supera el kilómetro de ancho, flanqueado a su vez, por barrancos que tienen aproximadamente entre 50 y 100 metros de altura (Rostain, 1999; Salazar, 2000). El río nace de las vertientes orientales de la cordillera Real, dominada en ese punto por el volcán Sangay. A pocos kilómetros de su nacimiento presenta una fuerte curva que dirige sus turbulentas aguas al sur, donde finalmente desemboca en el río Santiago.

El medio ambiente de la región es húmedo-tropical y se encuentra dentro de lo que se conoce como selva alta (aproximadamente 1200 m.s.n.m.). El clima no es tan caluroso como en la selva baja, pero presenta abundantes precipitaciones, especialmente, en los meses invernales. La gran diversidad ecológica responde a los diferentes rasgos geográficos que existen, tomando en cuenta la influencia de la cercanía tanto del pie de monte andino, como de la cordillera del Cutucú. La vegetación actualmente está dominada por grandes extensiones de pastizales dedicados a la ganadería sobre lo que tiempo atrás 
fue bosque. No obstante, también existen reductos de selva primaria y secundaria en la superficie de la llanura oriental, occidental y en franjas pequeñas de bosque en las riveras de los ríos. Además de los pastizales se identifican especies nativas de árboles como chontas, pambiles (Iriartea deltoides y Socratea sp.), cedros (Cedrela rosei), copales (Liquidambar styraciflua), balsas, algunas concentraciones de guadua (Guadua angustifolia), etc. La fauna, en cambio se caracteriza por la presencia de armadillo (Dasypus novemcinctus), guanta (Dasyprocta punctata), saíno (Tayassu peccari), tucán, pava de monte, varias especies de serpientes, además de tigrillo, puma (Felis Concolor), jaguar y venado (Odocoileus virginianus); estos últimos prácticamente desaparecidos en las áreas ahora deforestadas por la colonización.

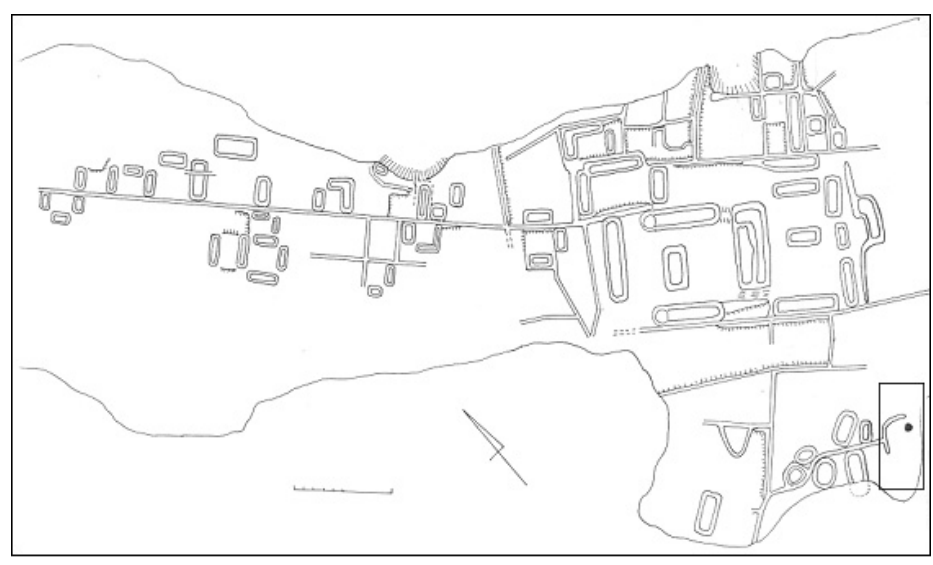

Este dibujo ha sido facilitado por Ernesto Salazar.
FIGURA 2.

Plano del complejo Huapula donde se distingue el conjunto La Lomita en el recuadro inferior derecho.

\section{Antecedentes}

La región del valle del río Upano fue conocida arqueológicamente luego de varios hallazgos tempranos. Una cerámica caracterizada principalmente por una decoración con bandas rojas entre incisiones fue reportada por Harner (1973), Bushnell (1946) y Lino Rampon (1959). Sin embargo, no es hasta varios años después que se inician los primeros trabajos en la región a cargo de Pedro Porras Garcés $(1985,1987)$. El escenario que encontró Porras sorprendió al mismo investigador: concentraciones de montículos artificiales de tierra y amplias muestras de material lítico y cerámico caracterizaban a los asentamientos prehispánicos de la zona. Porras se dedicó al estudio de la zona organizando varias temporadas de campo durante las cuales efectuó múltiples excavaciones, así como el levantamiento topográfico de las plataformas artificiales del recinto que bautizo como "Sangay" (actualmente renombrado como Huapula).

1 Porras denominó al recinto principal de sus investigaciones como "Sangay", dado que el volcán localizado al oeste del valle domina la vista de la cordillera. Porras creía que los grupos Upano adoraban al volcán por lo que orientaron sus montículos hacia el mismo. En la actualidad el proyecto Sangay-Upano renombró al sitio como Huapula, nombre del río que corre al este del recinto. Las nuevas investigaciones no han podido comprobar un culto al volcán; aunque sí se ha demostrado que las plataformas no se encuentran orientadas al nevado (ver Salazar, 2000). 
Con la información recolectada, la investigación de Porras se centró en dos problemas específicos: 1) establecer una tipología y seriación cerámica y 2) develar la función de las plataformas artificiales. En ambos casos su trabajo presentó algunos problemas generados, por un lado, por varias inconsistencias estratigráficas y de datación radiocarbónica; mientras que, por otro, los esfuerzos por explicar la función de las plataformas artificiales derivaron en la búsqueda de un patrón iconográfico en la forma de distribución espacial de los montículos en el sitio Huapula ${ }^{2}$. Tras la culminación de las investigaciones en el sector, las inquietudes respecto a la evolución cultural se tornaron aún mayores.

Una década más tarde, el proyecto arqueológico Sangay-Upano inició varios trabajos en la zona aportando con nueva información al localizar y registrar numerosos complejos a ambas riveras del río Upano (ver Salazar, 1998, 1999, 2000; Rostain, 1999a, 1999b, 1999c; ver también Ochoa, Rostain y Salazar, 1997). Estos estudios produjeron importantes datos sobre la construcción de caminos, plazas y plataformas, así como de un conjunto cerámico posteriormente definido como Huapula (Rostain, 1999a, 1999b, 1999c). Paralelamente a estos estudios, Arthur Rostoker (1996 y 2005) realizó varios trabajos en el sitio de "Yaunchu" localizado en las cercanías de Sucúa en la zona sur del valle, lugar en el que Harner años atrás reportó material Upano. Posteriormente, Stephen Rostain efectuó nuevas excavaciones en el sitio "Kilamope" a 9 km. al norte de Macas en la orilla izquierda del río Upano, bajo el proyecto Río Blanco. Durante sus investigaciones en este lugar Rostain encontró una cerámica distinta que denominó "Kilamope": “...caracterizada por incisiones, impresiones cordeladas y pintura negativa marrón y beige.” (Rostain, 2006:4). Sin embargo, aparte de esta descripción no existe mayor referencia sobre este material.

En la actualidad, la región del valle del Alto Upano está marcada por la ausencia de nuevas investigaciones arqueológicas. Por consiguiente, es evidente que pese a que todos los trabajos realizados en la zona han contribuido con valiosa información, los datos han permanecido aislados y demandan de estudios que permitan conectarlos desde una perspectiva regional. Por esto, el trabajo aquí expuesto pretende establecer un nuevo marco de discusión sobre el desenvolvimiento cultural en el valle a partir de la presentación de una secuencia cultural para el sitio La Lomita.

\section{Problema}

Las investigaciones de Porras, entre 1979 y 1989, se concentraron en el área del alto Upano, tras el descubrimiento de varios sitios con montículos artificiales. El equipo de investigación llevó a cabo varias excavaciones, cortes estratigráficos y recolecciones superficiales en diferentes zonas. Amplias muestras de material cerámico fueron obtenidas en las catorce temporadas de campo registradas dentro del proyecto. Dicho material fue

2 Sobre esto Porras (1987) realizó interpretaciones muy al azar, como por ejemplo el hecho de afirmar que la distribución de los montículos seguía un patrón iconográfico en el que supuestamente podía apreciarse la imagen de una hembra jaguar en trance de cópula con un hombre. Los trabajos últimos del proyecto Sangay- Upano, de acuerdo a los nuevos levantamientos topográficos, han desechado la idea de una iconografía presente en la distribución de los montículos del recinto Huapula (ver Salazar, 1999: 204-205). 
procesado y analizado dando lugar a la estructuración de una compleja tipología (fueron definidos 21 tipos cerámicos) a partir de la cual se estableció un cuadro cronológico compuesto por cuatro fases cerámicas: pre-Upano (2750-2520 a.C.), Upano I (1100-120 a.C.), Upano II (40 a.C. - 170 d.C.) y Upano III (170-940 d.C.). Así también Porras identificó un material distinto al que denominó como fase Chiguaza (Porras 1987, 256, 189-361). Los resultados fueron publicados en el libro Investigaciones Arqueológicas a las Faldas del Sangay.

Es precisamente una exhaustiva revisión de la publicación de Porras la que permitió encontrar algunas falencias en los criterios que condujeron la investigación. Las limitaciones de la metodología de clasificación cerámica empleada son evidentes cuando revisamos el número de tipos que identificó como Upano. En su análisis se distinguen dos errores que son fundamentales: 1) no se toma en cuenta que muchos atributos decorativos se combinan o cambian en relación con las formas del ceramio por lo cual no se pueden analizar por separado y 2) no se establece una división clara entre distintos estilos cerámicos, lo que genera a su vez varias inconsistencias en la cronología propuesta. Esto se debe principalmente a que Porras usó solamente los atributos decorativos de los fragmentos para armar la tipología. Esto produjo a la larga la definición de casi tantos tipos como atributos identificados. Por este motivo, diferenciar entre un estilo cerámico y otro se tornó complicado:

"Si se mide la identidad o similitud según el número de características comunes, sin tomar en cuenta ni la proporción de cada atributo dentro de la colección total, ni la manera en que se combinan los atributos, es posible (lógicamente) llegar a la conclusión de que dos estilos son idénticos cuando realmente no lo son" (Kay Tarble, 1982:18).

Probablemente por ello Porras no pudo distinguir la existencia de más de un conjunto cerámico en el material analizado. Pues, aunque es cierto que Porras menciona la existencia de un material pre-Upano, no distinguió las diferencias existentes entre estos conjuntos. Por ejemplo, cuando se refiere al pre-Upano describe una cerámica "burda con desgrasante ordinario o grueso" (Porras, 1987: 297), resaltando un contraste claro con la cerámica Upano. Sin embargo, si revisamos su tipología podemos encontrar que esta misma cerámica de característica "burda" y desgrasante grueso aparece en los tipos cerámicos Upano Inciso y Upano Aplique Sencillo y con Muescas; dejando así en claro ciertas falencias de procedimiento en el manejo cerámico que no han estado ausentes en arqueología. Es por ello que resulta confuso encontrar diferencias claras entre el material Pre-Upano y Upano (pues a ratos parecería que se refiere a ellos como un solo conjunto).

En concordancia con la cronología de Porras, el período pre-Upano presenta fechas tempranas que oscilan entre 2500 y 2000 a.C., lo que ubicaría a esta etapa dentro del período Formativo. Si le añadimos las fases Upano 1, 2 y 3, la secuencia de ocupación de Upano en el sector sería de aproximadamente 3000 años; y aun cuando, solamente tomáramos como punto de partida las fechas otorgadas a Upano 1 (1100-120 a.C.), estaríamos hablando de una cultura que se desarrolló desde el Formativo medio-tardío y prosiguió por aproximadamente 2000 años. Esto representa un lapso de tiempo extenso para una sola cultura de las características de Upano.

En los últimos años, una nueva cronología ha sido propuesta por Stephen Rostain (2006) basado en la información proveniente de sus investigaciones en Huapula. En 
esta se propone al menos dos ocupaciones: Upano y Huapula. Los grupos Upano de acuerdo a Rostain se habrían asentado en el valle entre el 700 a.C. y el 400 d.C., siendo remplazados tras su declive por la gente Huapula que ocupó la zona entre el 800 y 1200 d.C. ${ }^{3}$. Las diferencias con el modelo de Porras son claras, especialmente si se considera que la evidencia arqueológica refuerza el modelo expuesto por Rostain (este modelo se lo analizará con mayor detenimiento más adelante). Esto permitió establecer algunas pautas sobre las que se guió el presente análisis.

\section{Análisis cerámico y secuencia cultural en el sitio La Lomita}

El sitio La Lomita, se encuentra ubicado en sector oeste del recinto Huapula (ver figura 1), en una pequeña planicie que termina en una depresión que luego desciende abruptamente al barranco del río Upano. De acuerdo a la descripción de Salazar (1996: 24), en esta parte se encuentran pequeños montículos y un camino corto que conecta hacia la depresión. Este sitio fue objeto de varias excavaciones estratigráficas en el lugar de un basural prehispánico ubicado a pocos metros del filo del barranco. El sitio fue seleccionado por la alta concentración de tiestos obtenidos en los sondeos previos realizados en la zona. La intención fue obtener datos concretos de un contexto de basural que permita construir una tipología y cronología cerámica. En efecto, en las excavaciones se recuperó una amplia colección cerámica, en cuyo análisis se encuentra basado el trabajo aquí expuesto.

El análisis de la cerámica proveniente del sitio la Lomita se efectuó como parte de mi disertación de licenciatura (Pazmiño, 2008). En este estudio se cumplió con dos objetivos principales: 1) depurar la tipología cerámica existente, y 2) establecer una cronología cerámica para el sitio La Lomita.

La estructuración de una tipología a partir de la cerámica del sitio La Lomita, permitió depurar la tipología existente hasta entonces para el área. Así, en la colección cerámica de La Lomita se distinguieron los siguientes tipos: Sangay Lóbulos Incisos, Sangay Aplique, Upano Ordinario, Upano Inciso, Upano Rojo entre Incisiones, Upano Pintura Roja, Upano Incisión Roja, Upano Blanco y Rojo, Upano Negativo, y Huapula Corrugado. Conjuntamente, una revisión de pastas y formas de un considerable número de fragmentos y una vasija (que había sido previamente reconstruida y restaurada), determinó la presencia de cerámica Cosanga en contexto Upano, reconociendo la existencia de al menos cinco recipientes distintos. Esta nueva tipología confirmó la existencia de tres conjuntos cerámicos distintos con los que se estructuró una secuencia cultural para el sitio.

Desde la publicación del libro Investigaciones Arqueológicas a las Faldas del Sangay de Porras (1987), en el que presenta un secuencia cronológica para los asentamientos Upano, pocos han sido los trabajos que se han interesado en abordar el tema cronológico en la región. Una de las principales investigaciones que ofreció valiosos aportes a este tema fue el trabajo de Rostain (1999a, 1999b, 1999c, 2006) tanto como parte del proyecto

3 Adicionalmente, en la misma publicación, Rostain señala la existencia de otro conjunto cerámico distinto de los anteriores denominado "Kilamope". No obstante, la falta de mayores evidencias no permite discernir de manera explícita si se trata de un conjunto distinto o una cerámica característica de una fase tardía de Upano (2006:4). 
arqueológico Sangay-Upano, como del posterior proyecto Río Blanco. Las excavaciones efectuadas por este investigador desenterraron un nuevo conjunto cerámico, de filiación tardía en relación al material Upano. Estas evidencias no sólo apoyaron la idea sobre la diversidad de asentamientos en el valle, sino ofrecieron indicios sobre las distintas ocupaciones del mismo cronológicamente.

Partiendo de ello, Rostain (1999a, 1999b, 1999c, 2006) pudo esclarecer de mejor manera la secuencia cultural de la zona mediante el análisis de la evidencia recuperada de una de las plataformas artificiales de Huapula. Las excavaciones produjeron datos sobre dos asentamientos diferentes, claramente perceptibles en el montículo. El primero en directa relación a los asentamientos Upano, mientras que el segundo se trató de una ocupación doméstica tardía a la que se denominó Huapula. Las fechas más recientes publicadas por Rostain (2006) establecen la ocupación Upano entre el 700 a.C. y el 400 d.C., y la ocupación Huapula entre el 800 y 1200 d.C. Al parecer, según este autor, los grupos Huapula llegaron al valle luego de que una erupción volcánica del Sangay habría puesto fin a la ocupación Upano. De acuerdo con Rostain (1999b, 1999c, 2006), estos grupos al establecer sus asentamientos habrían reutilizado los montículos. El contexto doméstico de una casa Huapula excavado en la "tola central" apoya esta idea; no obstante, de todos los montículos excavados es la única evidencia de reutilización reportada hasta el momento ${ }^{4}$.

Con respecto a la ocupación Upano, mientras que Porras la estimó en tres fases ${ }^{5}$, Rostain (1999b, 65-66) por su parte prefiere hablar de la existencia de al menos dos fases. Para ello se apoya en las excavaciones que efectuó en la llamada "tola central", la misma que presenta evidencias de una primera etapa ocupacional Upano en la capa basal de la plataforma. Esto sería un indicio de que la "tola" habría sido levantada posteriormente en el transcurso de una segunda etapa caracterizada por la construcción de montículos. Al respecto cabe señalar que, aunque Porras establece para la fase Upano 1 (1200-120 a.C.), un período demasiado largo de tiempo, la fecha en la que señala habría comenzado la construcción de los montículos, es decir hacia el 200 a.C., es mucho más aceptada (ver Rostain 1999b). Esto nos muestra que ambos investigadores coinciden en cuanto a la diferenciación de dos momentos Upano: uno previo a la construcción de las plataformas artificiales y otro que identifica el momento mismo de la construcción. Recientemente Rostain hace referencia a un nuevo conjunto cultural más temprano que la ocupación Upano. Este investigador señala en la información dispuesta en la página web del proyecto Río Blanco ${ }^{4}$, la existencia de un nuevo material cerámico al que denomina Sangay, relacionado con grupos que al parecer ocuparon la zona hacia el 700 a.C., aunque no esclarece el período que habría durado esta ocupación.

La cronología de Rostain establece de esta manera, una nueva secuencia cronológica en la que se identifican por el momento tres etapas culturales distintas: Sangay hacia 700

4 Incluso me atrevería a decir que es la única evidencia concreta de ocupación prehispánica sobre un montículo de la región. Esto resulta extraño; ya que, tanto Rostain como Salazar atribuyen un carácter ocupacional a los montículos. No obstante, hasta la fecha no se ha podido identificar todavía sobre los montículos ningún contexto habitacional Upano. 
a.C.; Upano entre el 700 a.C. y 400 d.C. y Huapula entre el 800 y 1200 d.C. Las notables diferencias que exhiben los restos cerámicos entre sí, constituyen por sí mismas un claro indicio de la presencia de distintos conjuntos cerámicos en el sitio de la investigación. A mi parecer esto representa un buen punto de partida para el establecimiento de una cronología cerámica que dilucide la correspondencia temporal de los artefactos del sitio La Lomita.

Precisamente, el objetivo del análisis fue encontrar una conexión entre la distribución de los tipos cerámicos en la estratigrafía y determinar cómo varían las formas cerámicas en la misma. A partir de ello se logró establecer una relación concreta entre la aparición o desaparición de tipos y la variación de las formas. Debido a que el mayor volumen de material se concentró en la cerámica Upano, se consideró pertinente establecer en primera instancia las correlaciones entre los tipos cerámicos pertenecientes a este conjunto; ya que, de esta manera, las comparaciones con los otros conjuntos se pudieron establecer de mejor forma.

El análisis del material Upano con respecto de la estratigrafía, permitió distinguir algunas características interesantes:

- La proporción de restos cerámicos se incrementó considerablemente en los estratos superiores.

- Los tipos Upano Negativo y Upano Blanco y Rojo mostraron una corta y bien marcada presencia en la estratigrafía; por el contrario de los otros tipos Upano que se encuentran presentes en casi toda la estratigrafía.

- Las formas de fuentes también presentan una corta y marcada presencia en los estratos superiores junto con los tipos arriba mencionados.

- La proporción de vajilla de servir y contener se incrementó en los estratos superiores.

Dos momentos pueden encontrarse representados por la cerámica Upano de La Lomita. El primero que podría marcar los inicios del asentamiento Upano en el valle reflejado en la discreta proporción de restos cerámicos, con un mayor predominio de la decoración de pintura roja entre incisiones; y un segundo momento caracterizado por un incremento significativo en la vajilla cerámica con la inclusión de nuevos motivos decorativos como el negativo y la pintura blanca y roja, además de una nueva forma cerámica representada por el aparecimiento de las fuentes.

Una vez esbozadas ciertas características principales perceptibles en el conjunto Upano, se comparó la distribución de la cerámica Sangay y la cerámica Huapula con respecto a la ocupación principal correspondiente a Upano. Por un lado fue evidente la concentración de los escasos fragmentos Huapula en los niveles superiores; mientras que por otro, la cerámica Sangay se ubica en mayor cantidad en los niveles inferiores, aunque aparece junto con algunos pocos restos cerámicos Upano. Esta correspondencia de la cerámica Sangay con parte de la cerámica Upano puede deberse a una mezcla de los depósitos, producto de la primera ocupación Upano. Para ello fue necesario analizar la naturaleza de los estratos de La Lomita.

Al revisar cuidadosamente los registros estratigráficos del lugar, se aprecia la presencia casi continua de dos estratos en los que se concentró el material cerámico. Cada capa presenta ciertas particularidades que vale la pena rescatar. El estrato más profundo (capa III) es un depósito de tierra amarilla en el que se destaca la presencia de ceraturos 
dispersos en todo el estrato. En algunas cuadrículas se identificó una subdivisión de este depósito en dos capas (capa IIIa y IIIb). Ambas capas están conformadas por tierra amarilla con impurezas, aunque la del fondo estaría conformada por tierra mucho más uniforme y compacta. Al parecer, la consistencia en general del depósito (especialmente el IIIa) con algunas intrusiones y una presencia no uniforme de ceraturos sugiere que fue alterado, muy probablemente, durante una primera ocupación Upano explicando así la correspondencia estratigráfica ocasional de materiales Sangay y materiales Upano. En cuanto al segundo estrato (capa II), éste presenta mayor uniformidad en cuanto a su constitución, algo que se manifiesta en cómo en este estrato se recuperó la mayor cantidad de restos cerámicos y líticos. Esta capa evidenciaría la ocupación principal Upano; ya que a este estrato corresponden generalmente los niveles donde se concentra la mayor cantidad de material recuperado.

\begin{tabular}{|c|c|c|c|c|}
\hline \# & Laboratorio & Proveniencia & Fechas BP & Calibración \\
\hline 1 & Beta -89267 & $\begin{array}{c}\text { Plataforma 4, } 260 \mathrm{~cm} . \mathrm{b} / \mathrm{s} \text {, nivel basal; Dataría } \\
\text { construcción de plataforma }\end{array}$ & $2160+/-80$ & 375BC-AD 65 \\
\hline 2 & Beta -90630 & $\begin{array}{c}\text { Subcomplejo XI, montículo central, } \\
160 \mathrm{~cm} . \mathrm{b} / \mathrm{s} \text {, nivel basal; dataría construcción } \\
\text { de montículo }\end{array}$ & $1790+/-60$ & AD 100-405 \\
\hline 3 & Beta -89269 & $\begin{array}{l}\text { Camino, Zanja } 1,140 \mathrm{~cm} . \mathrm{b} / \mathrm{s} \text {, dataría } \\
\text { construcción del mismo }\end{array}$ & $1650+/-120$ & AD 160-665 \\
\hline 4 & Beta -89270 & $\begin{array}{c}\text { Plataforma 5, NW/T1, cuadrícula 3, } 305 \mathrm{~cm} \text {. } \\
\text { b/d, nivel basal; dataría construcción de } \\
\text { plataforma }\end{array}$ & $2310+/-70$ & $\begin{array}{c}515 \\
\text { BC- } 190 \mathrm{BC}\end{array}$ \\
\hline 5 & Beta -89271 & $\begin{array}{l}\text { Plataforma 5, NW/T1, cuadrícula 3, } 256 \mathrm{~cm} \text {. } \\
\text { b/d, nivel intermedio; dataría una segunda fase } \\
\text { de construcción del montículo, i.e. la } \\
\text { acumulación de ceraturo amarillo }\end{array}$ & $2780+/-90$ & $\begin{array}{c}1115 \\
\text { BC-785 BC }\end{array}$ \\
\hline 6 & Beta -100305 & Sitio Lomita, 1N1W, Lg. 70/Lt. 90, 51 cm. & $1070+/-90$ & $\mathrm{AD} 780-1175$ \\
\hline 7 & Beta -100306 & $\begin{array}{l}\text { Sitio Lomita, 1N2W, Lg. 54/Lt. 16, } 201 \mathrm{~cm} \text {; } \\
\text { 1N2W, Lg. 66/Lt. 70, } 107 \mathrm{~cm} \mathrm{b/d}\end{array}$ & $1510+/-60$ & $\mathrm{AD} 425-655$ \\
\hline 8 & Beta -100307 & $\begin{array}{c}\text { Sitio Lomita, 1N2W, Lg. 11/Lt. 100, } \\
\text { 100-109 cm; 2N2W, Lg. 24-61/Lt. 48-79, } \\
\text { 200-206 cm b/d }\end{array}$ & $1990+/-70$ & $\begin{array}{c}165 \\
\text { BC-AD } 160\end{array}$ \\
\hline 9 & Beta -100308 & Subcomplejo XI, montículo central & $940+/-60$ & AD 995-1235 \\
\hline 10 & Beta -100309 & Subcomplejo XI, montículo central & $2110+/-70$ & 365 BC-AD 55 \\
\hline
\end{tabular}

Cuadro proporcionado por Ernesto Salazar.

Tres muestras de carbón fueron recuperados de estos estratos; una perteneciente al nivel 21 localizado en el fondo del tercer estrato, otra perteneciente al nivel 11 ubicado en la parte inferior del segundo estrato intermedio y, finalmente, una tercera muestra obtenida entre los niveles 5 y 6 en el primer estrato. Aunque tres muestras es un número bastante limitado para establecer una adecuada correlación temporal del depósito de La 
Lomita, considero que la existencia de otros fechamientos radiocarbónicos obtenidos en Huapula permite esbozar una secuencia temporal para los asentamientos de la zona. Las fechas radiocarbónicas obtenidas de estas muestras provenientes de La Lomita (ver cuadro 1, muestras 6, 7 y 8 correspondientes a La Lomita) presentan algunas novedades que cabe revisar. Cada una de estas fechas se encuentra en perfecta secuencia con la estratigrafía del lugar. Así, se distingue que la muestra obtenida en el nivel 21, estrato inferior, arrojó una fecha comprendida entre el 165 a.C. - 160 d.C., en tanto la muestra del nivel 10 perteneciente a la capa intermedia produjo una fecha entre el $425-655$ d.C. y finalmente la fecha del estrato superior (nivel 5) oscila entre $780-1175$ d.C..

Si observamos detenidamente las fechas de La Lomita, la más antigua, correspondiente a 165 a.C. - 160 d.C., es una fecha bastante tardía si consideramos el inicio de la ocupación Upano en el 700 a.C.. Si apreciamos el cuadro de fechas presentado por Rostain (1999b: 89), vemos que al parecer este autor fechó el inicio de la ocupación Upano en 700 a.C., valiéndose apenas de una muestra radiocarbónica. Lo extraño del caso es que el mencionado investigador obtuvo una fecha del nivel basal de la "tola central", datada entre el 365 a.C. - 55 d. C.. A ello adherimos otra datación obtenida, de acuerdo a Rostain, de una muestra en la base del montículo 4, fechada entre el 375 a.C - 65 d.C.. Más aún en la explicación de las fechas señala que: "En la Tola Central del Complejo XI, la capa basal Upano está fechada entre 365 AC y 405 DC (4 muestras)" (Rostain, 1999b: 89). Es notorio lo tardío de estas fechas, especialmente si tomamos en cuenta que corresponden a un estrato que representaría el momento previo o inicial de la construcción del montículo. Otras fechas obtenidas de los niveles basales de los montículos arrojan fechas mucho más tardías aún. Por ejemplo, encontramos tres fechas que van entre 75 a.C. - 120 d.C.; 75 330 d.C.; y 100 - 405 d.C. (Rostain, 1999b: 89). Esta revisión de las fechas obtenidas de contextos estratigráficos en el sitio de Huapula, sugiere que la presencia Upano en el valle es mucho más tardía, probablemente hacia el 380 a.C.. Incluso la correspondencia de las fechas podría señalar por lo menos dos períodos bien definidos: una primera ocupación entre el 380 a.C. - 65 d.C.; que marcaría la llegada de los grupos Upano al valle donde habrían comenzado sus primeros asentamientos; seguida de una etapa entre 65 - 400 d.C., donde se produciría un incremento de su poder en el valle, caracterizado por la construcción de montículos.

Consecuentemente, es probable que el fenómeno de los montículos sea más reciente en la región; pues la mayor parte de fechas sugieren la construcción tardía de los montículos. Si retomamos las fechas obtenidas en La Lomita muy bien podríamos afirmar que la primera fecha corresponde al período inicial de la ocupación Upano, reflejado en las características de la evidencia cerámica encontrada en este nivel. Así también, aunque la fecha obtenida de una muestra de la segunda capa es mucho más tardía, podría muy bien expresar la etapa de construcción de montículos. La fecha más tardía de La Lomita, obtenida del nivel superior, encaja perfectamente en el período establecido por Rostain para la ocupación Huapula ubicada entre el 700 y 1200 d.C..

Una vez realizadas todas las correspondencias, notamos en primera instancia la presencia de tres conjuntos cerámicos distintos que sugieren tres ocupaciones de diferente naturaleza en el lugar. Segundo, vemos que el aumento de la cantidad de fragmentos Upano se encuentra en relación al aumento de las formas reconstruidas. Al mismo tiempo notamos que en el momento que ocurre un aumento en el número de fragmentos 
y recipientes, ocurren dos eventos significativos. Por un lado aparecen de repente dos tipos cerámicos como lo son el Upano Negativo y el Upano Blanco y Rojo; y por otro lado, al mismo tiempo, surge una nueva forma cerámica representada por las fuentes. Si a esto añadimos las fechas de C-14 obtenidas tanto en el yacimiento de La Lomita como en varios sectores del complejo Huapula, vemos que los rasgos presentes en la cerámica Upano podrían estar en correspondencia con dos momentos centrales en el desarrollo de este pueblo: el proceso inicial de asentamiento en el valle; seguido de un período de construcción de montículos. En este sentido podríamos resumir una secuencia cultural en la que se distinguen tres grupos con diferente naturaleza que se asentaron en la región durante períodos distintos:

1. Ocupación Sangay: la ocupación Sangay está caracterizada principalmente por una cerámica muy particular, en la que destaca el desgrasante grueso, la decoración incisa y el aplique. Los grupos pertenecientes a la sociedad Sangay habrían habitado el valle probablemente entre el 900 y 500 a.C. Pese a la escasa información obtenida de este tipo de ocupación podemos destacar que los detalles en la cerámica expresan una filiación muy representativa de los grupos amazónicos. Porras (1987) reporta cerámica con estos rasgos en los poblados de Chiguaza y Paulo VI, hacia el sector norte del valle. No obstante, Rostoker (1996, 2005), en sus trabajos en Sucua, en el sur del valle, no ha reportado material semejante; lo que probablemente define el área de asentamientos Sangay entre el río Palora y el cauce alto del río Upano. Tanto las formas de las vasijas como los motivos decorativos están muy vinculados a rasgos netamente amazónicos. Por las características de la evidencia reducida especulamos en que la ocupación Sangay fue dispersa y probablemente de bajo índice poblacional. Esto habría cambiado drásticamente con la llegada de los grupos Upanos al valle.

FIGURA 3.

Olla reconstruida del tipo Sangay Lóbulos Incisos.

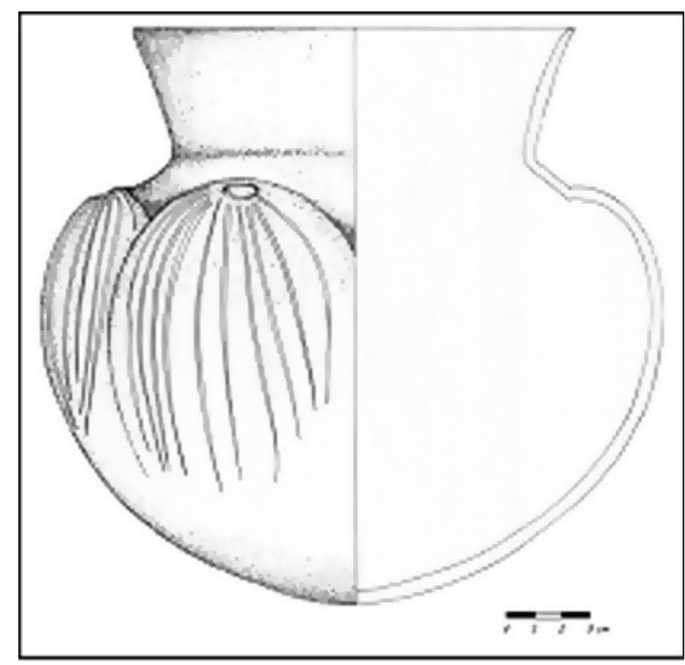

2. Ocupación Upano: los grupos de la tradición Upano probablemente se asentaron en el valle alrededor del 380 a.C.. La llegada de estos grupos a una zona estratégica por su ubicación para el intercambio comercial, sin duda habría generado cambios significativos en la región. Es posible que tras ir consolidando su dominio en el valle, paulatinamente incrementaron los contactos comerciales con el área andina. Al parecer en este período en varios lugares de la frontera andino-amazónica se vive un momento de contacto intenso. La primera fase de Upano se habría desarrollado entre el 380 a. C. y el 65 d.C., en la 
cual se inicia el intercambio comercial con la sierra. Característica de este período es la presencia mayoritaria de la cerámica del tipo Upano Rojo entre Incisiones, aunque también se encuentran presentes los tipos Upano Inciso, Upano pintura Roja y Upano Ordinario. La segunda fase Upano habría estado caracterizada por el inicio de las construcciones de montículos probablemente hacia el 65 d.C., en este período se registra el aparecimiento de la decoración negativa y la pintura blanca y roja en los cuencos, así como también la incorporación de una nueva forma cerámica: las fuentes. Como resultado de las tareas de construcción de montículos se habría incrementado significativamente el número de la vajilla cerámica para servir alimentos; así como también las dimensiones de los recipientes donde probablemente se contenía la bebida. Esto lo podemos evidenciar directamente en el incremento del material cerámico de La Lomita, especialmente vinculado a recipientes de almacenar y servir. De acuerdo a Rostain (1999b, 2006) las evidencias de una capa de ceniza volcánica reflejarían que la ocupación Upano habría desaparecido por una erupción del volcán Sangay. El fin de la ocupación Upano habría tenido lugar alrededor del 400 d.C..

FIGURA 4.

Olla Upano Rojo entre Incisiones

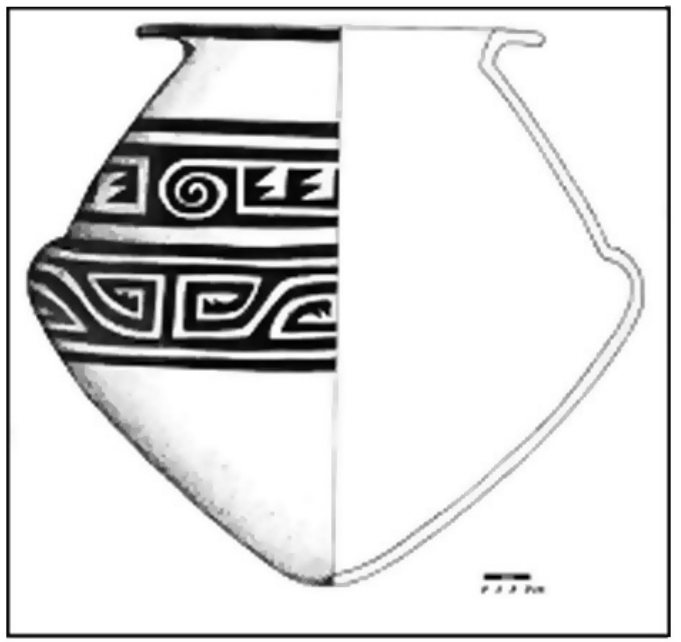

3. Ocupación Huapula: según Rostain (2006), luego de un período de intensa actividad volcánica del Sangay, el valle habría sido abandonado para siglos más tarde ser reocupado por los grupos Huapula entre el 700 y 1200 d.C.. La comparación etnográfica de un contexto doméstico y las viviendas Achuar de la amazonía ecuatoriana dejan entrever que los grupos Huapula no habrían sido muy distintos a los grupos Achuar contemporáneos. De filiación y características netamente amazónicas, los nuevos ocupantes del valle aprovecharon algunos de los montículos dejados por los Upanos siglos antes. La evidencia material se encuentra caracterizada por la presencia de la decoración corrugada en vasijas amplias probablemente usadas para contener chicha; así como en metates y manos de moler. Las características de los contextos excavados presentan elementos domésticos que nos conducen a pensar que la ocupación Huapula fue dispersa y no tuvo la fuerza de la anterior ocupación. Estos grupos habrían llegado a la zona alrededor del 700 d.C. y su ocupación pudo haberse mantenido hasta el 1200 d.C.

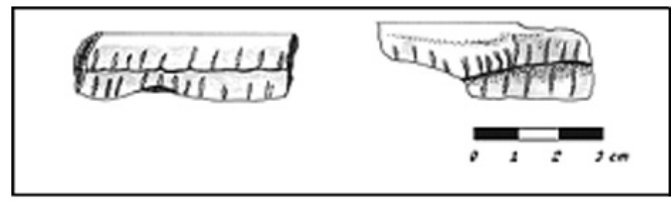

FIGURA 5. Bordes Huapula Corrugado 


\title{
Discusión
}

Lahabitación del valle refleja episodios concretos en los que se produjeron asentamientos con influencias estilísticas particulares. Más aún, su ubicación en las inmediaciones de la cordillera establece un vínculo entre las regiones andina y amazónica que no se puede pasar por alto. En la arqueología ecuatoriana este problema no ha sido lo suficientemente discutido y pocos trabajos analizan las evidencias de esta conexión (Bruhns, Burton y Rostoker, 1994; Bray, 1996). Es necesario abordar con mayor profundidad las influencias que ejercieron entre sí dos sistemas culturales distintos. Las particularidades de cada uno de ellos los convierten en sistemas fuertes que, sin duda, ejercieron presión en las áreas limítrofes provocando constantes intercambios de información. Precisamente, el valle del alto Upano (considerado como selva alta) presenta las condiciones ideales, dada su estratégica ubicación, para explorar las formas de interacción entre estas dos regiones en la época prehispánica.

Uno de los trabajos que examina algunas pruebas de este contacto es el presentado por Bruhns, Burton y Rostoker (1994). En él analizan concretamente el caso de la cerámica de bandas rojas entre incisiones encontradas en yacimientos de la sierra. De acuerdo a este estudio, los resultados del análisis de las pastas realizado a este material determinan que la arcilla no es local y su fuente se localiza en las estribaciones orientales cercanas al volcán Sangay. Es decir, el origen de esta cerámica de bandas rojas entre incisiones encontrada en la sierra, pertenecería a la región del Alto Upano. De ser este el caso, los nexos de la sociedad Upano con la sierra podrían haber comenzado al menos entre el 300 a.C.. Esta información correspondería con el comienzo de la ocupación Upano estimada aproximadamente entre el 380 a.C.. Por lo tanto, el predominio del tipo cerámico Upano Rojo entre Incisiones sobre otros tipos en esta etapa puede muy bien verse reflejado en su aparecimiento en la sierra.

\begin{abstract}
"Uno de los primeros materiales foráneos notado en las excavaciones en Pirincay fue la cerámica IFR (Inciso Franjas Rojas). Fue por primera vez que se encontró dentro de un buen contexto estratigráfico. Sin excepción, la cerámica IFR se encuentra en las capas superiores del basural, asociada con material pertinente a la ocupación tardía de Pirincay. Esta fase fue fechada por medio de C14 aproximadamente entre 400 a.C.-100 d.C.. Es así como en esta época podemos distinguir algunos cambios dentro de la red de intercambio a larga distancia. Los artefactos costeños: cuentas de Spondylus, cerámica chorreroide y conchas del mar, se hallaron en cantidades menores o desaparecieron. A la vez, el intercambio con las culturas peruanas creció y tenemos evidencia de pastoreo de llamas, del crecimiento de las artes textiles, del conocimiento del uso de metales (cobre, cobre dorado, oro fundido) y el patrón centro-andino de las fiestas en las cuales se halló el consumo de carne de llamas y una bebida (¿chicha?), la disposición ritual de los restos animales, y las ollas y copas rotas en pozos especiales" (Bruhns et al., 1994:58).
\end{abstract}

Bruhns, Burton y Rostoker señalan además, que el aparecimiento del Rojo entre Incisiones coincide con un momento en el que los contactos comerciales con la costa se ven disminuidos; abriendo esta situación claramente las oportunidades de comercio con la región amazónica. Bajo estas circunstancias se abre la posibilidad para pensar que los grupos Upano empezaron un intercambio comercial con la región interandina 
desde sus inicios. De esta manera se podría entender que el crecimiento paulatino de la actividad comercial produjera su afianzamiento en la región; pues cabe suponer que un incremento en el comercio pudo coadyuvar a generar recursos suficientes que respalden el crecimiento económico y por consiguiente la construcción de montículos.

FIGURA 6.

Olla filiación Cosanga

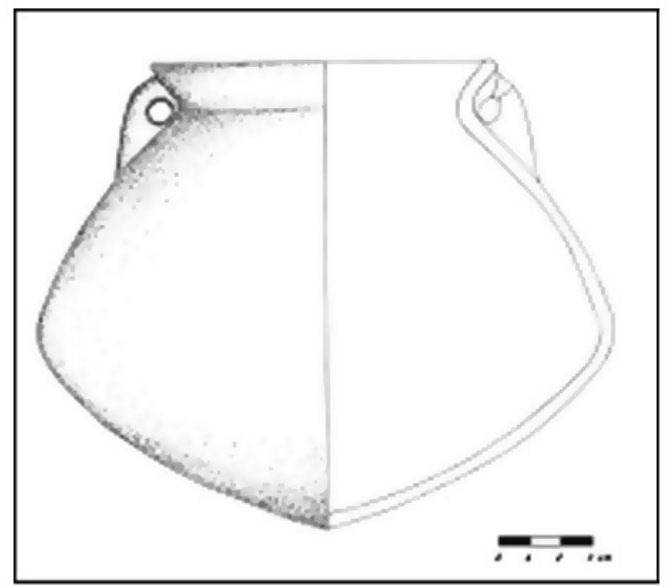

Las evidencias de cerámica de origen Cosanga sugieren claramente que su llegada fue producto de un contacto comercial, o bien con los grupos asentados hacia el norte en la ceja de montaña y piedemonte oriental, o por medio de un contacto con la sierra. De cualquier modo el hallazgo de restos de esta cerámica asociada a los niveles profundos, permite ver que los grupos Upano para entonces no fueron los únicos en extender sus contactos con la región interandina, puesto que, hacia el norte en la zona de los valles de Cosanga, Baeza y Quijos, los grupos Cosanga ya habían estructurado una red comercial con los pueblos de la sierra. Ya anteriormente la investigación llevada a cabo por Tamara Bray (1996) sacó a relucir que la cerámica Cosanga (Panzaleo) fue producida en la amazonía y que la presencia de ella en contextos serranos obedece a dinámicas de intercambio. Bray al revisar algunas fechas tempranas para esta cerámica termina por aceptar una distribución cronológica que ubica la presencia de esta cerámica en contextos serranos entre el 300 a.C. y el 1550 d.C.. Este fechamiento temprano se encuentra en relación con la fecha estimada del surgimiento de la sociedad Upano. La aparición de esta cerámica en el contexto de la Lomita no sólo es un indicio de que esta cerámica constituía ya un objeto de intercambio, sino que puede tal vez ayudarnos a explicar la presencia de cerámica Upano en contextos serranos como el de Pirincay. Pues, es posible que la gente Upano haya tratado de involucrarse en un modelo comercial parecido al de los Cosanga, especialmente dada la necesidad de afianzar las redes de intercambio. Podríamos pensar entonces, que al poder establecerse dentro de una red de intercambio con los pueblos interandinos, seguramente obtuvieron los beneficios necesarios que permitieron financiar la construcción de montículos artificiales y consolidarse en la región.

En los tres momentos de ocupación del valle se perciben claras diferencias entre las vajillas usadas en cada cultura. Tanto la cerámica Sangay como la Huapula presentan formas y decoraciones muy comunes en la región amazónica. Por el contrario, la cerámica Upano aunque mantiene algunas formas que se encuentran con frecuencia en otras áreas de la amazonía, incorpora otras tantas más relacionadas con los grupos serranos. A ello se suma la presencia de la decoración negativa muy frecuente en la sierra y costa. 
Por otro lado, la construcción de montículos artificiales conservando algunos patrones hasta cierto punto geométricos, sugiere una influencia que pudo bien llegar desde la sierra o incluso desde la costa. Pues, es evidente que el fenómeno de montículos entre los Upano aparece tardíamente. Es decir, luego de un considerable lapso de tiempo de mantener contacto con la sierra:

"Las influencias de la sierra se hallan presentes en la edificación de montículos artificiales de tierra, la difusión de cerámicas en el seno de una extensa red comercial, el uso de metates de piedra pulida y de grandes ollas para preparar cerveza de maíz. Por otra parte, se pueden apreciar numerosas características de las culturas selváticas amazónicas, entre ellas la ubicación de los sitios a orillas de un río, la técnica de fabricación y el decorado de la cerámica" (Rostain, 1999b: 59).

A ello se puede añadir dos eventos que ocurren en la segunda fase de Upano y se perciben en el material cerámico. Por un lado hay un notable incremento de la vajilla cerámica, especialmente de cuencos y ollas; y por otro se percibe un incremento en el tamaño de los recipientes de manera considerable. Este aumento del tamaño y cantidad de los recipientes se asocia a la vajilla de almacenar y servir. Consecuentemente este fenómeno podría bien reflejar la organización de fiestas comunales con ocasión de la construcción de montículos. Pues, no resultan ajenas las prácticas en las que algunos pueblos prehispánicos con ocasión de la construcción de algún montículo o estructura importante, organicen fiestas en las que se distribuye comida y bebida con el fin de fortalecer la imagen de reciprocidad entre el pueblo y sus gobernantes.

En fin, la evidencia fruto del análisis del material cerámico de La Lomita permite establecer algunas correlaciones con la finalidad de reorientar la visión sobre el desarrollo cultural en el valle del Alto Upano. Cabe, sin embargo, resaltar que las intenciones del presente trabajo han sido poner un poco más de luz sobre el desenvolvimiento cultural prehispánico de los sociedades amazónicas a partir del análisis del material cerámico del sitio La Lomita; aunque es claro que todavía quedan muchas interrogantes por explorar con respecto al tema.

\section{Conclusiones}

Luego de varios años de incertidumbre con respecto a la tipología y cronología establecida por Porras para la tradición Upano; el hallazgo de evidencias de la ocupación tardía Huapula permitió a Stephen Rostain vislumbrar la posibilidad de la existencia de otras ocupaciones en el valle, lo que le llevó a establecer un nuevo cuadro cronológico (Rostain 1999b, 2006). Parte de los planteamientos de Rostain han podido ser corroborados en el presente trabajo.

El estudio de la tipología y la construcción de una cronología cerámica para el sitio La Lomita redireccionan las visiones sobre el desenvolvimiento cultural prehispánico en el valle del Alto Upano, al establecer la presencia de tres ocupaciones culturales diferentes, representadas por los conjuntos cerámicos Sangay, Upano y Huapula.

La evidencia de restos de una ocupación temprana identificada como "Sangay" presenta características de pasta, forma y decoración que están guiadas por técnicas y estilos muy propios de la región amazónica. La presencia de vasijas con formas y estilos 
decorativos clásicos de las tierras bajas son elementos de juicio que permiten identificar a la vajilla Sangay con un estilo netamente amazónico.

Así también, encontramos una cerámica caracterizada por una decoración con pintura roja entre incisiones, que marca la ocupación Upano. Esta ocupación, presenta evidencia de al menos dos fases culturales. Una primera fase que marca el inicio de la ocupación del valle entre el 380 a.C. y el 55 d.C., seguida de una segunda fase en la que tiene lugar la construcción de montículos entre el 50 d.C. y el 400 d.C..

Paralelamente podemos señalar que el conjunto cultural Upano presenta algunos elementos no comunes en la región amazónica, además de algunos utensilios cerámicos importados desde otras áreas. Por ejemplo, la presencia de cerámica con bandas rojas entre incisiones en algunos lugares de la sierra, así como la recurrencia de decoración negativa, además de cerámica no local del estilo Cosanga reflejan los contactos con otras regiones.

Por otra parte, la evidencia, aunque exigua, de fragmentos de cerámica Huapula en los niveles superficiales de La Lomita, permitió corroborar la ocupación tardía de la zona por parte de los grupos Huapula; cuestión que ha sido fuertemente evidenciada anteriormente por Rostain (1999a, 1999b, 1999c, 2006).

Finalmente, es claro que el valle del Alto Upano fue escenario de un complejo desarrollo social prehispánico. Diferentes culturas en distintas épocas ocuparon el valle, dejando algunas pistas sobre su paso. La intención, precisamente de este trabajo ha sido presentar desde una perspectiva diacrónica, a partir del análisis de la cerámica del sitio La Lomita, los procesos culturales ocurridos en un sitio en particular, con la finalidad de que sirvan a su vez de referencia y comparación para el entendimiento del desarrollo cultural acaecido en la región.

\section{Bibliografía}

Bray, Tamara L., 1996, "El Problema Panzaleo : Una Cerámica No-Local en la Sierra Norte del Ecuador", en: Langebaek, Carl y Felipe, Cárdenas Arroyo (eds.), Caciques, Intercambio y Poder: Interacción Regional en el Área Intermedia de las Américas, Universidad de Los Andes, Bogotá.

Bruhns, Karen et al., 1994, "La Cerámica « Incisa en Franjas Rojas »: Evidencia de Intercambio entre la Sierra y el Oriente en el Formativo Tardío del Ecuador", en: Izumi, Shimada (ed.), Tecnología y Organización de la Producción Cerámica Prehispánica en los Andes, Pontificia Universidad Católica del Perú, Fondo Editorial, Perú.

Bushnell G., 1946, "An Archaeological Collection from Macas, on the Eastern Slopes of the Ecuadorian Andes", Man, 46(2):2-6.

Harner, Michael, 1973, The Shuar, people of the sacred waterfalls, Anchor Books, New York.

Ochoa, Myriam et al., 1997, "Montículos Precolombinos en el Alto Upano", en: Revista Cultura, № 2: 54.

Pazmiño, Estanislao, 2008, Análisis Cerámico del Sitio La Lomita, Morona Santiago, Ecuador. Disertación de grado, Pontificia Universidad Católica del Ecuador, Quito.

Porras, Pedro I., 1987, Investigaciones Arqueológicas a las Faldas del Sangay, Artes Gráficas Señal, Quito.

1985a, Arte Rupestre del Alto Napo-Valle del Misagualli, Ecuador, Artes Gráficas Señal, Quito.

1985b, "Archaeological Investigation of the Sangay Site, Ecuador", National Geographic Society, Research Reports. Vol. 21. Washington D.C..

1978, Arqueología de la Cueva de los Tayos, Ediciones Universidad Católica, Quito.

1975a, Fase Cosanga, Ediciones Universidad Católica, Quito. 
1975b, Fase Pastaza: El Formativo en el Oriente Ecuatoriano, Separata de la revista de la Universidad Católica $\mathrm{N}^{\mathrm{a}} 10$, Quito.

Rampon, Lino, 1959, Sitio Arqueológico F.P. Cuadernos de Investigaciones Científicas \#1, Arqueología. Misiones Católicas de la Amazonía, Quito.

Rostain, Stéphen, 2006, "Etnoarqueología de las casas Hupaula y Jíbaro", Bulletin de l'Institut Français d'Etudes Andines, vol 35 (3), Lima, pp. 1-10.

1999a, Excavación en área en un montículo de Huapula, Amazonía Ecuatoriana (proyecto Sangay-Upano), en: Salazar, Ernesto (comp.), Memorias de Primer Congreso Ecuatoriano de Antropología vol 3, Museo Jacinto Jijón Y Caamaño, Departamento de Antropología PUCE, MARKA, Quito, pp. 227-256.

1999b, "Secuencia Arqueológica en Montículos del Valle del Upano en la Amazonía Ecuatoriana", en: Bulletin de l'Institute Fraçais d'Études Andines 28 (1), Lima, 53-89.

1999 c, "Occupations humaines et fonction domestique de monticules préhistoriques d'Amazonie équatorienne", Société Suisse des Américanistes Bulletin 63 : 71-95.

Rostoker, Arthur, 2005, Dimension of Prehistoric Human Occupation in the Southern Ecuadorian Oriente. Dissertation submitted to graduate Faculty of Anthropology in partial fulfilment of the requirements for the degree of Doctor in Philosopy, City University of New York.

1996, An Archaeological Collection from Eastern Ecuador. Thesis submitted to the Faculty of San Francisco State University in partial fulfilment of the requirements for the degree Masters of Arts in Anthropology.

Salazar, Ernesto, 2000, Pasado Precolombino de Morona Santiago. Macas, Casa de la Cultura Ecuatoriana Núcleo de Morona Santiago, Ilustre Municipio del Cantón Morona.

1999, De Vuelta al Sangay: Investigaciones Arqueológicas en el Alto Upano, en: Salazar, Ernesto (comp.), Memorias del Primer Congreso Ecuatoriano de Antropología vol. 3, Museo Jacinto Jijón y Caamaño, Departamento de Antropología PUCE, MARKA, Quito, pp. 183-225.

1998, "Naturaleza y Distribución de los Montículos Precolombinos de la Cuenca del Alto Upano, Ecuador", en: Cárdenas, Felipe, Tamara L. Bray, (ed.), Intercambio y Comercio entre Costa, Andes y Selva: Arqueología y Etnohistoria de Suramérica, Departamento de Antropología, Universidad de Los Andes, Bogotá.

1996, Investigaciones Arqueológicas en el curso superior del río Upano, Provincia de Morona Santiago. Informe preliminar presentado al Instituto Nacional de Patrimonio Cultural, Quito.

Saulieu, Geoffroy de y Lino Rampón Z., 2006, Colección Arqueológica de Morona-Santiago, AbyaYala, Quito. 